\title{
Recent results from Daya Bay
}

\author{
Ming-chung Chua ${ }^{1, *}$, on behalf of the Daya Bay Collaboration \\ ${ }^{1}$ Department of Physics, The Chinese University of Hong Kong, Shatin, N. T., Hong Kong
}

\begin{abstract}
Utilizing powerful nuclear reactors as antineutrino sources, high mountains to provide ample shielding from cosmic rays in the vicinity, and functionally identical detectors with large target volume for near-far relative measurement, the Daya Bay Reactor Neutrino Experiment has achieved unprecedented precision in measuring the neutrino mixing angle $\theta_{13}$ and the neutrino mass squared difference $\mid \Delta \mathrm{m}_{\text {ee }}^{2}$. I will report the latest Daya Bay results on neutrino oscillations and light sterile neutrino search.
\end{abstract}

\section{Introduction}

Neutrino flavor oscillations are caused by the misalignment between neutrino flavor and mass eigenstates, parameterized by mixing angles $\theta_{12}$ and $\theta_{23}$, and they are realized as the changing of neutrino flavors as a neutrino propagates freely. In 2012, the Daya Bay Reactor Neutrino Experiment discovered a non-zero value of $\sin ^{2} 2 \theta_{13}$ by observing the disappearance of reactor antineutrinos over kilometer distances [1], and in 2014, it reported the first measurement of the effective mass splitting $\left|\Delta \mathrm{m}^{2}{ }_{\text {ee }}\right|$ [2] by neutrino spectrum distortion [3]. In this talk, I will briefly review the Daya Bay experiment and present the latest results on neutrino oscillations and light sterile neutrino search.

\section{The Daya Bay Reactor Neutrino Experiment}

There are three underground experimental halls (EHs) - two near (EH1, EH2) and one far (EH3) - in the Daya Bay experiment [4], each hosting functionally identical, three-zone antineutrino detectors (ADs) - 2 in EH1 and EH2 each, 4 in EH3 - in a pool of ultrapure water segmented into the inner water shield (IWS) and outer water shield (OWS), both instrumented with photomultiplier tubes (PMTs). The water pool and Resistive Plate Chambers (RPCs) placed above it are muon veto systems, and the water buffer also serves to suppress radiation from the surrounding rocks. See Fig. 1 and 2.

\footnotetext{
* Corresponding author: mcchu@phy.cuhk.edu.hk
} 


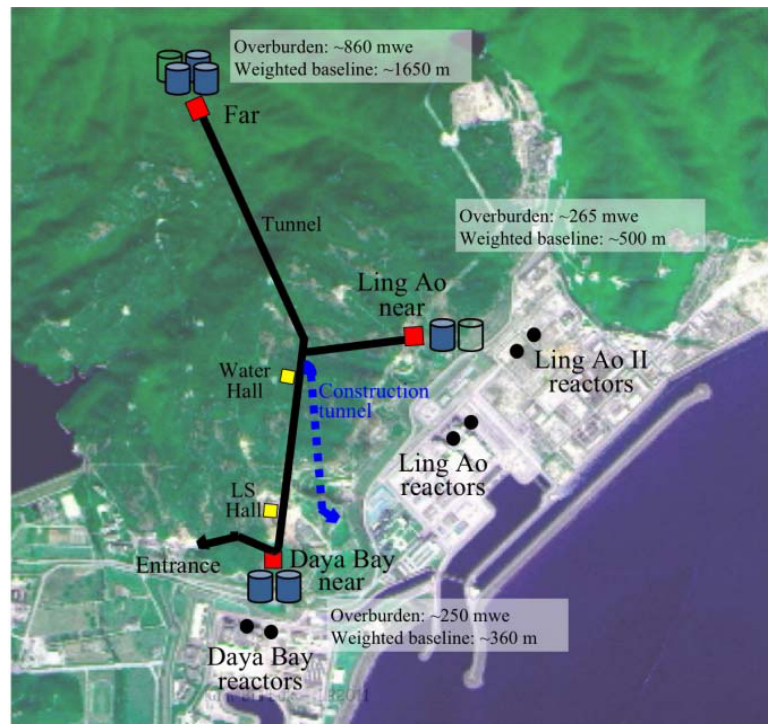

Figure 1. The layout of the Daya Bay Experiment.

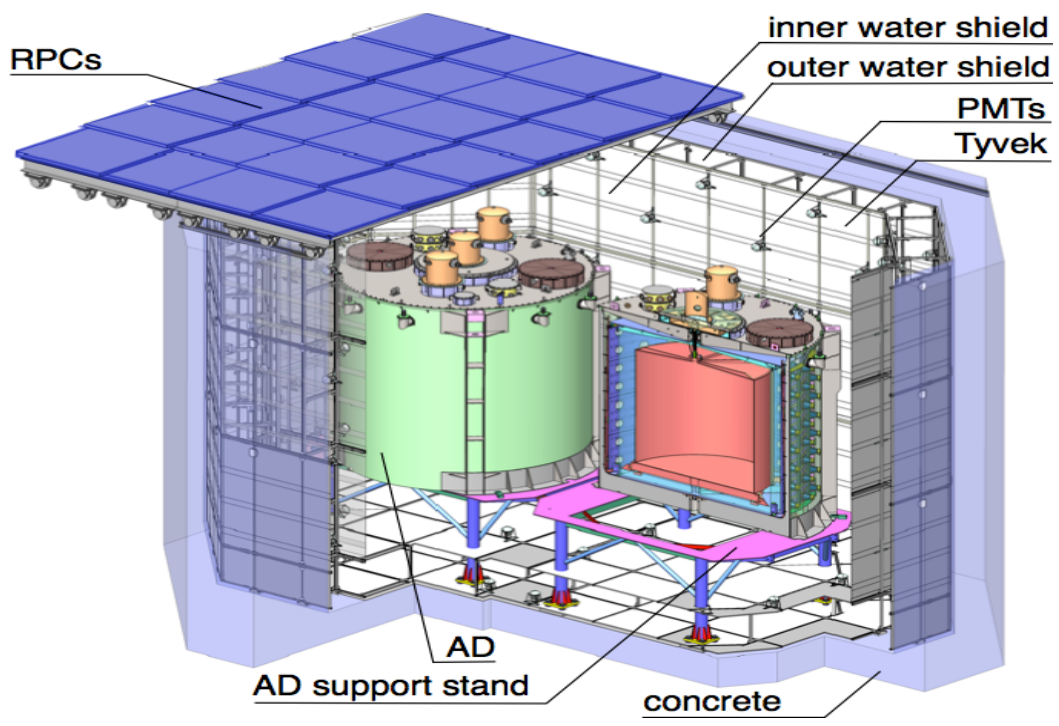

Figure 2. A water pool in each EH hosts 2 (4) ADs for measuring antineutrinos from the reactors. Adapted from [4]. 
Each AD contains 20 tons of gadolinium-doped liquid scintillator (LS) in the inner acrylic vessel (AV), 20 tons of undoped LS in the outer AV and 37 tons of mineral oil which buffers the 192 radially positioned 20-cm PMTs in the outermost steel tank [5]. The ADs have a light yield of $\sim 165$ photoelectrons/ $\mathrm{MeV}$ and a reconstructed energy resolution of $\sigma_{E} / E \approx 8 \%$ at $1 \mathrm{MeV}$.

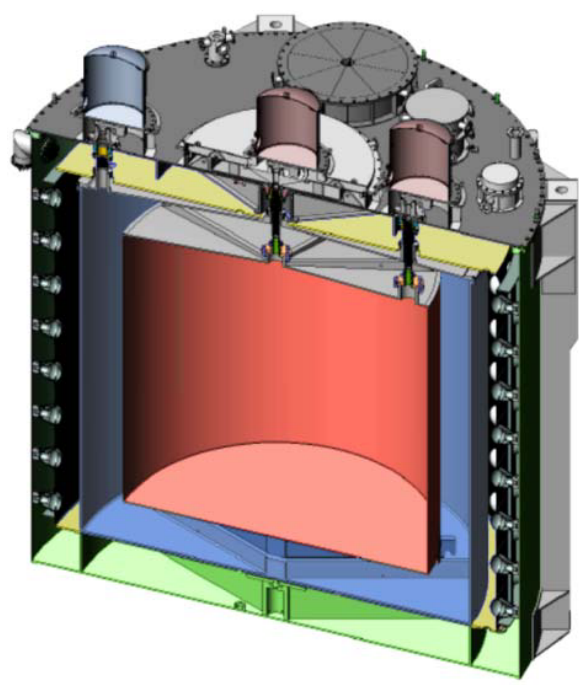

Figure 3. Each AD contains 3 zones of liquids and 192 PMTs to detect the light generated by particle interactions. Adapted from [5].

Reactor antineutrinos are detected via the inverse $\beta$-decay (IBD) reaction, $\bar{v}_{\mathrm{e}}+\mathrm{p} \rightarrow \mathrm{e}^{+}+\mathrm{n}$. The energy of the prompt light emitted by the positron $E_{\text {prompt }}$ is directly related to the neutrino energy: $E_{\bar{v}}=E_{\text {prompt }}+E_{\mathrm{n}}+0.78 \mathrm{MeV}$, where $E_{\mathrm{n}}$ is the average neutron recoil energy $\sim 10 \mathrm{keV}$. The neutron is captured by Gadolinium doped into the liquid scintillator in the inner acrylic vessel, with a mean capture time of $\sim 30 \mu \mathrm{s}$, emitting gamma rays (totaling $\sim 8 \mathrm{MeV}$ ) as a delayed signal. The requirement of prompt and delayed coincidence greatly suppresses background. The use of relative measurement by functionally identical ADs in the near and far experimental halls leads to large suppression of systematic uncertainties. Detailed discussion of the IBD selection, efficiencies, backgrounds and systematic uncertainties can be found in [4].

Fig. 4 shows the operation history of the Daya Bay experiment, number of IBD events accumulated in the near and far detectors and the measured value of $\sin ^{2} 2 \theta_{13}$ as a function of time. The experiment will continue operation until 2017, by the end of which more than 4 million IBD events will be recorded.

\section{Oscillation results}

The survival probability of electron antineutrinos is given by

$P_{\mathrm{ee}}=1-\sin ^{2} 2 \theta_{13} \sin ^{2}\left(\Delta m_{\mathrm{ee}}^{2} L / 4 E\right)-\sin ^{2} 2 \theta_{12} \cos ^{4} \theta_{13} \sin ^{2}\left(\Delta m_{21}{ }^{2} L / 4 E\right)$,

where $E$ is the neutrino energy and $L$ the distance between the neutrino source and detection. 


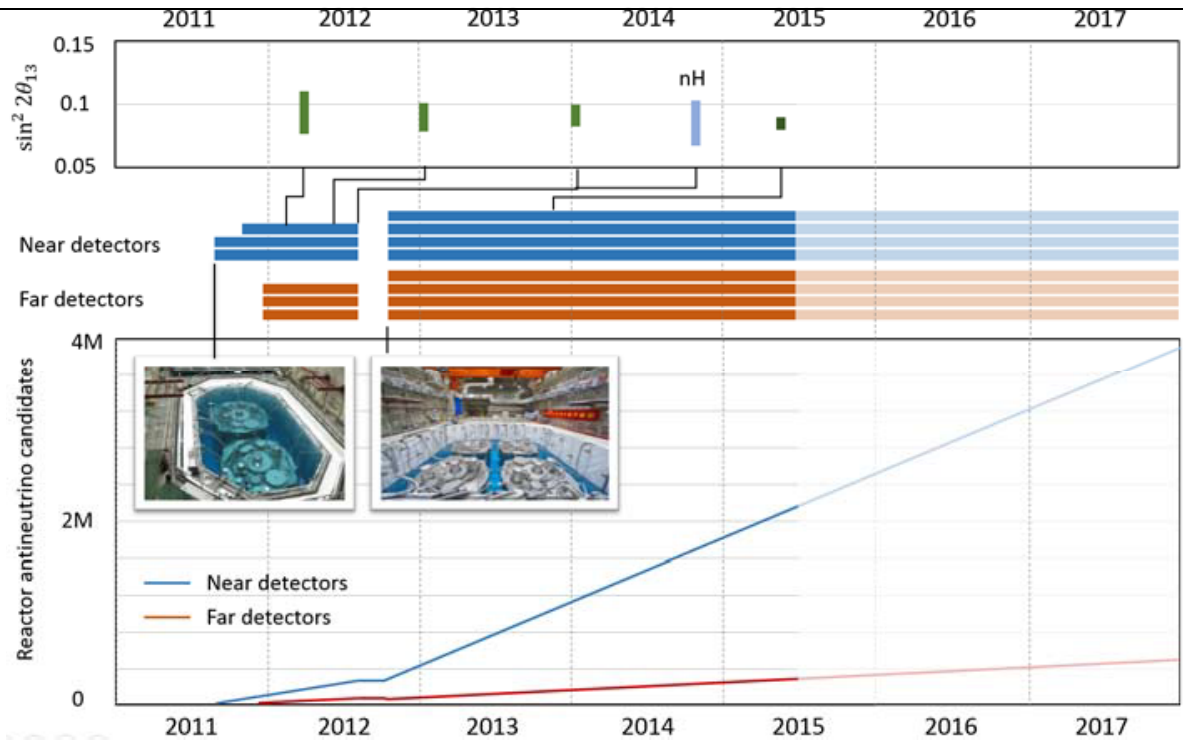

Figure 4. Operation history of the Daya Bay Experiment and the measured values of $\sin ^{2} 2 \theta_{13}$.

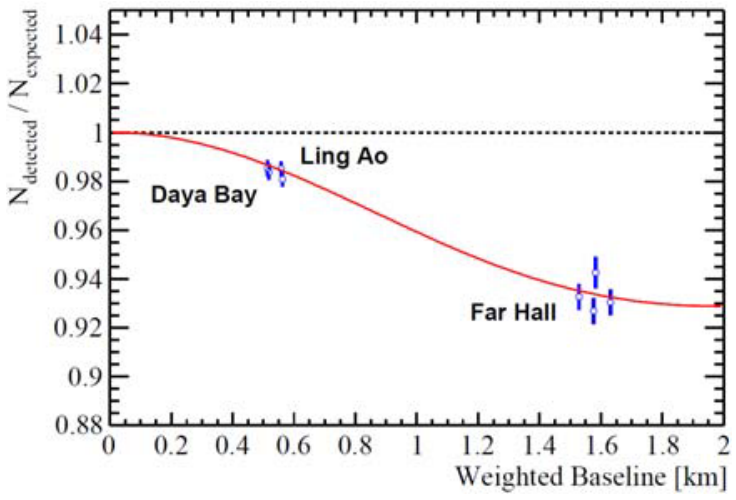

Figure 5. Measured deficit of the neutrino flux as a function of baseline, compared to the survival probability with best-fit result for $\sin ^{2} 2 \theta_{13}$. Adapted from [6].

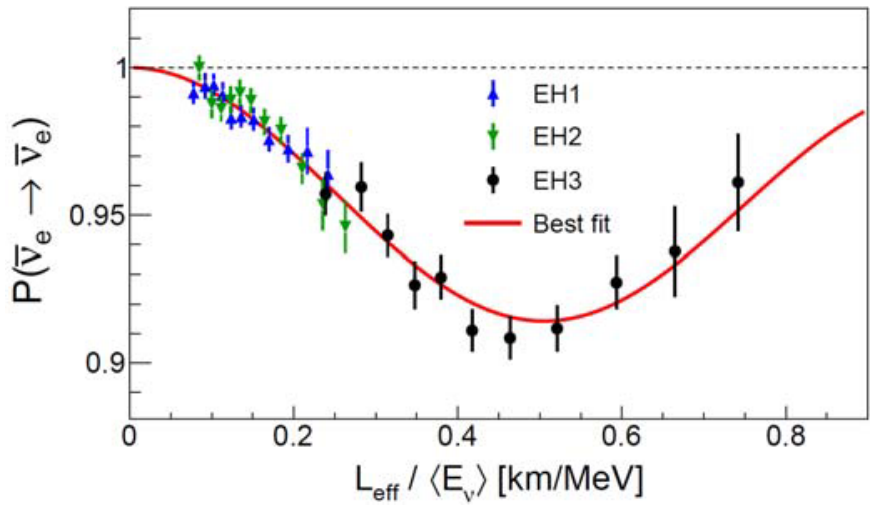

Figure 6. Distortion of neutrino energy spectrum as a function of $L / E$, compared to calculation of survival probability using the best-fit value of $\sin _{4}^{2} 2 \theta_{13}$. Adapted from [6]. 
The neutrino mixing angle $\theta_{13}$ can therefore be measured by the deficit of the energyintegrated neutrino flux at far ADs using the near ADs as reference (the rate method), or the distortion of the neutrino energy spectrum (the rate-plus-shape method).

Figure 5 shows the rate deficit of all 8 ADs in near and far experimental halls of Daya Bay [6], whereas Figure 6 shows the spectral distortion measured at Daya Bay [6]. The results of the neutrino oscillation parameter fitting are shown in Figure $7 . \sin ^{2} 2 \theta_{13}$ is determined to be $0.084 \pm 0.005$ by Daya Bay with an unprecedentedly high precision of about $6 \%$. An independent measurement using $\mathrm{nH}$ captures, which are characterized by larger target mass but more background contamination yields $\sin ^{2} 2 \theta_{13}=0.083 \pm 0.018$ [7]. Furthermore, $\left|\Delta m^{2}{ }_{\text {ee }}\right|$ is measured to be $(2.42 \pm 0.11) \times 10^{-3} \mathrm{eV}^{2}$, which agrees with results by muon neutrino disappearance experiments MINOS [8] and T2K [9], with comparable precision.

\section{Search for a light sterile neutrino}

A popular candidate for nonbaryonic dark matter [10] is sterile neutrinos with $\sim \mathrm{keV}$ masses. An ev-mass-scale sterile neutrino was introduced [11] to resolve two well-known tensions between cosmic microwave background (CMB) and local measurements of the Hubble constant $H_{o}$ and the amount of structures [12], as well as between CMB and lensing measurements [13]. The extra energy density of the sterile neutrinos increases the expansion rate of the universe at recombination and thus changes the acoustic scale, and their free streaming suppress small structures today. If light sterile neutrinos mix with the three active neutrinos, they could be detected via modification of the oscillation patterns of active neutrinos. The standard $3 \times 3$ neutrino mixing matrix would become a $4 \times 4$ matrix, with new mixing angles such as $\theta_{14}$ entering the survival probability of electron antineutrinos.

The Daya Bay Experiment search for a light sterile neutrino via its mixing with the active neutrinos using more than 300,000 reactor antineutrino interactions collected during the six-detector data period from December 2011 to July 2012 [14]. Using a relative measurement from its two near halls, Daya Bay is most sensitive to active-sterile neutrino mixing in the $10^{-3} \mathrm{eV}^{2}<\left|\Delta m^{2}\right|<0.3 \mathrm{eV}^{2}$ range. We look for an additional spectral distortion with a frequency different from that of the atmospheric mass splitting. 


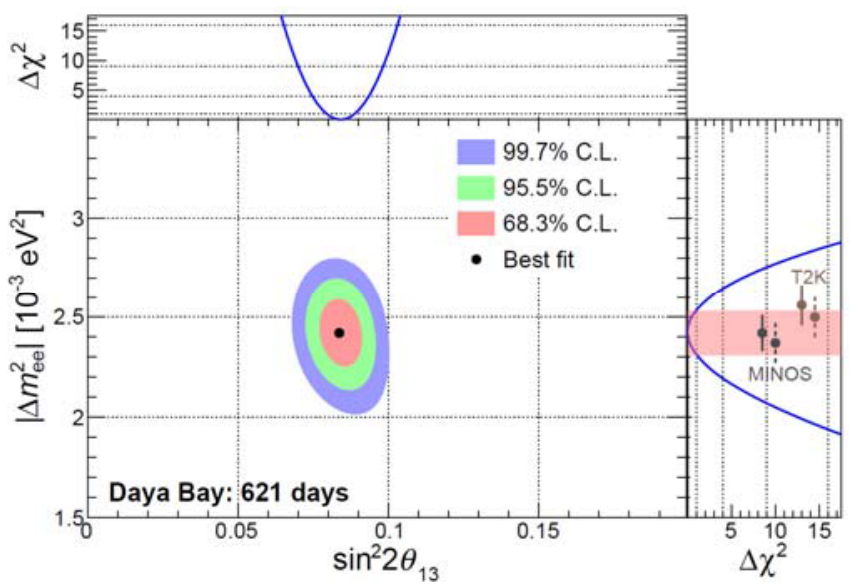

Figure 7. Rate-plus-shape results for $\mid \Delta m^{2}$ ee $\mid$ and $\sin ^{2} 2 \theta_{13}$. Adapted from [6].

Figure 8 shows a comparison between the Daya Bay prompt energy spectra in EH2 and $\mathrm{EH} 3$ and the expected ones based on EH1 spectra if a light sterile neutrino exists with $\left|\Delta m^{2}{ }_{41}\right|=4 \times 10^{-3} \mathrm{eV}^{2}$ and $4 \times 10^{-2} \mathrm{eV}^{2}$, assuming $\sin ^{2} 2 \theta_{14}=0.1$. The Daya Bay data are consistent with the standard 3-flavor prediction taking into account experimental uncertainties. Exclusion of a region in the $\left|\Delta m^{2}{ }_{41}\right|$ vs. $\sin ^{2} 2 \theta_{14}$ parameter space is achieved,

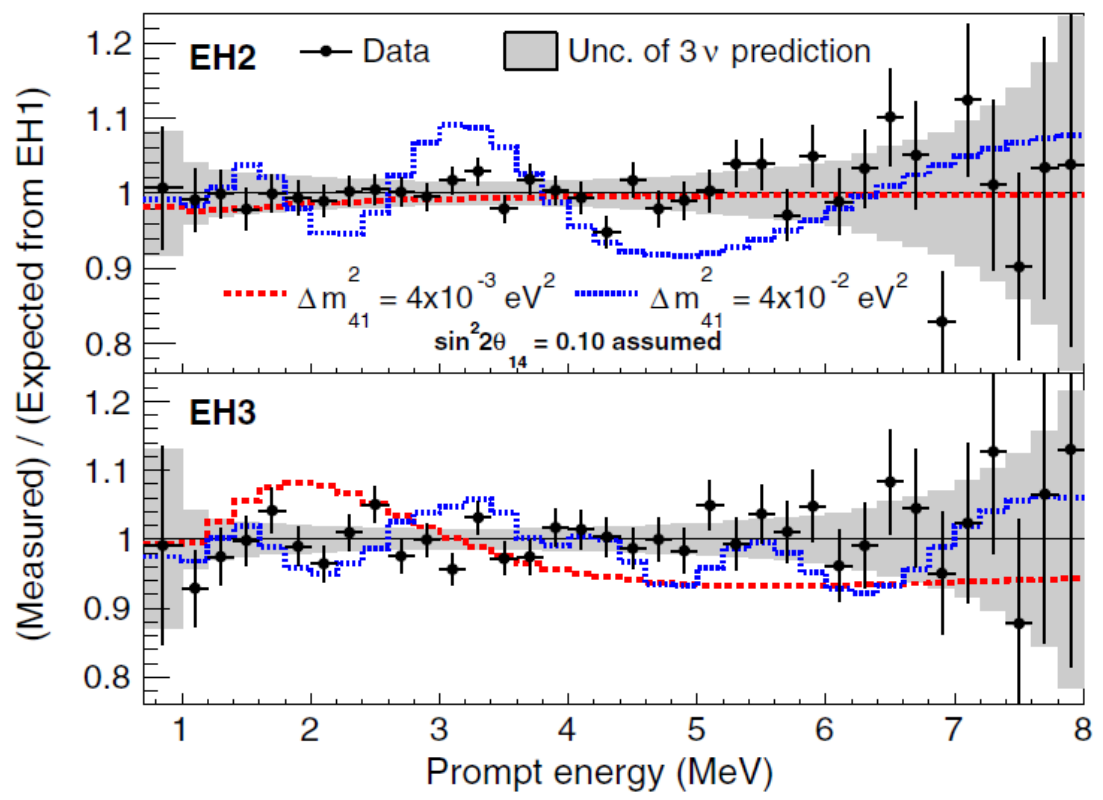

Figure 8. Measured prompt energy spectrum in EH2 and EH3 compared to expectation based on EH1 data, if a light sterile neturino exists with two values of $\left|\Delta m^{2}{ }_{41}\right|$ and $\sin ^{2} 2 \theta_{14}=0.1$. Adapted from [14]. 
shown in Figure 9. Daya Bay is uniquely sensitive to the $10^{-3} \mathrm{eV}^{2}<\left|\Delta m^{2}{ }_{41}\right|<0.3 \mathrm{eV}^{2}$ range [14], which was largely unexplored previously.

\section{Summary}

The Daya Bay Reactor Neutrino Experiment has achieved unprecedented precision in measuring the neutrino mixing angle $\theta_{13}$ and the neutrino mass squared difference $\mid \Delta m^{2}$ ee $\mid$, using relative measurement by near and far detectors. Making use of several different baselines provided by the three experimental halls, the Daya Bay experiment excluded a light sterile neutrino in the $10^{-3} \mathrm{eV}^{2}<\left|\Delta m^{2}{ }_{41}\right|<0.3 \mathrm{eV}^{2}$ range. The experiment has accumulated a large set of neutrino events, allowing for more searches for non-standard neutrino interactions and physics beyond the standard model.

\section{Acknowledgements}

The Daya Bay experiment is supported in part by the Ministry of Science and Technology of China, the United States Department of Energy, the Chinese Academy of Sciences, the National Natural Science Foundation of China, the Guangdong provincial government, the Shenzhen Municipal government, the China Guangdong Nuclear Power Group, Shanghai Laboratory for particle physics and cosmology, the Research Grants Council of the Hong Kong Special Administrative Region of China, the focused investment scheme of CUHK and University Development Fund of The University of Hong Kong, the MOE program for Research of Excellence at National Taiwan University and NSC funding support from Taiwan, the U.S. National Science Foundation, the Alfred P. Sloan Foundation, the University of Wisconsin, the Virginia Polytechnic Institute and State University, Princeton University, California Institute of Technology, University of California at Berkeley, the Ministry of Education, Youth and Sports of the Czech Republic, the Joint Institute of Nuclear Research in Dubna, Russia, and the NSFC-RFBR joint research program. We are grateful for the ongoing cooperation from the China Guangdong Nuclear Power Group and China Light \& Power Company. 


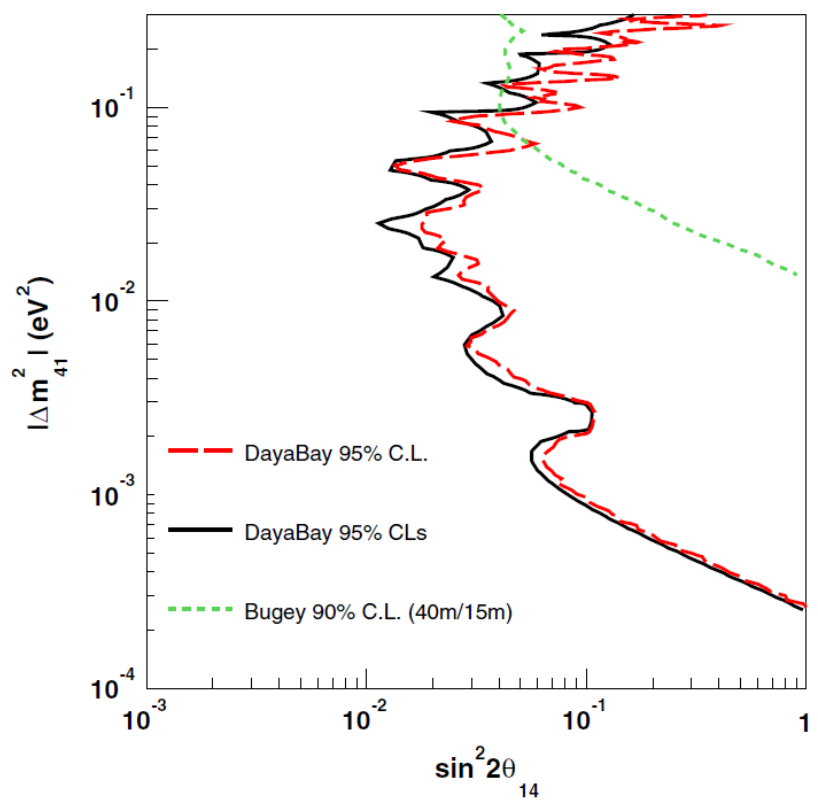

Figure 9. Exclusion plot for $\left|\Delta m^{2}{ }_{41}\right|$ and $\sin ^{2} 2 \theta_{14}$. Parameter space on the right of the curves are excluded at $95 \%$ C. L. The Daya Bay result is complementary to Bugey's result and is uniquely sensitive to the $10^{-3} \mathrm{eV}^{2}<\left|\Delta m^{2}{ }_{41}\right|<0.3 \mathrm{eV}^{2}$ range. Adapted from [14].

\section{References}

[1] F. P. An et al. (Daya Bay Collaboration), Phys. Rev. Lett. 108, 171803 (2012); Chin. Phys. C37, 011001 (2013); Phys. Rev. D90, 071101 (2014).

[2] $\Delta m^{2}$ ee is an effective mass splitting that can be obtained by replacing $\cos ^{2} \theta_{12} \sin ^{2} \Delta_{31}+$ $\sin ^{2} \theta_{12} \sin ^{2} \Delta_{32}$ with $\sin ^{2} \Delta_{\text {ee }}$, where $\Delta_{j i}=1.267 \Delta m_{j i}^{2}\left(\mathrm{eV}^{2}\right)[L(\mathrm{~m}) / E(\mathrm{MeV})]$, and $\Delta m_{j i}^{2}=m_{j}^{2}$ $m_{i}{ }^{2}$ is the difference between the mass-squares of the mass eigenstates $v_{\mathrm{j}}$ and $v_{\mathrm{i}}$. To estimate the values of $\Delta m^{2}{ }_{31}$ and $\Delta m^{2}{ }_{32}$ from the measured value of $\Delta m^{2}$ ee, See Supplemental Material at http://link.aps.org/supplemental/10.1103/PhysRevLett.115.111802 .

[3] F. P. An et al. (Daya Bay Collaboration), Phys. Rev. Lett. 112, 061801 (2014).

[4] F. P. An et al. (Daya Bay Collaboration), Nucl. Instrum. Methods Phys. Res., Sect. A 685, 78 (2012).

[5] H. Band et al., JINST 8, T11006 (2013).

[6] F P. An et al. (Daya Bay Collaboration), Phys. Rev. Lett. 115, 111802 (2015).

[7] F P. An et al. (Daya Bay Collaboration), Phys. Rev. D 90, 071101 (2014).

[8] P. Adamson et al. (MINOS Collaboration), Phys. Rev. Lett. 112, 191801 (2014).

[9] K. Abe et al. (T2K Collaboration), Phys. Rev. Lett. 112, 181801(2014).

[10] S. Dodelson and L. M. Widrow, Phys. Rev. Lett. 72, 17(1994); A. Kusenko, Phys.

Rep. 481, 1 (2009).

[11] Mark Wyman, Douglas H. Rudd, R. Ali Vanderveld, and Wayne Hu, Phys.

Rev.Lett.112, 051302 (2014).

[12] A. G. Riess et al., Astrophy. J. 730, 119 (2011); A. G. Riess et al., Astrophy. J. 732, 129 (2011).

[13] R. A. Battye and A. Moss, Phys. Rev. Lett. 112, 051303 (2014).

[14] F P. An et al. (Daya Bay Collaboration), Phys. Rev. Lett. 113, 141802 (2014). 